\title{
Tunable Wavelength-Demultiplexer by Tapered Photonic Crystal Waveguide
}

\author{
Zeki Hayran' ${ }^{1}$, Mirbek Turduev', Muriel Botey ${ }^{2}$, Ramon Herrero ${ }^{2}$, Kestutis Staliunas ${ }^{2,3}$, and Hamza Kurt ${ }^{1}$ \\ ${ }^{I}$ Nanophotonics Research Laboratory, Department of Electrical and Electronics Engineering, \\ TOBB University of Economics and Technology, Ankara 06560, Turkey \\ ${ }^{2}$ DONLL, Departament de Fisica i Enginyeria Nuclear, Universitat Politècnica de Catalunya (UPC), \\ Edifici Gaia, Rambla de Sant Nebridi 22, 08222 Terrassa, Spain \\ ${ }^{3}$ Institucio Catalana de Recercai Estudis Avanc, ats (ICREA), \\ Passeig Lluis Companys 23, 08010 Barcelona, Spain \\ e-mail:zekihayran.etu@gmail.com
}

\begin{abstract}
We numerically investigate the design of a wavelength de-multiplexer based on a tapered photonic crystal waveguide. The tapered waveguide is generated by reducing the width of the channel which, in turn, provides a gradual change in the effective index for the guided modes. Depending on the wavelength, the grading effect enables the propagating beam to be trapped at different spatial positions along the waveguide. Furthermore, alternation on the tapering angle, i.e. changing the slope of the tapered waveguide, will result in a spatial shifting of the trapping locations. Thus, the structure can be adjusted to pick up the frequencies of interest at the chosen positions. Numerical results show that, by placing vertical line defects as drop channels at specific locations, different wavelengths can be properly guided along the drop channels that are transverse to the main waveguide. Keywords: photonic crystals, tapered waveguide, wavelength demultiplexing, rainbow trapping.
\end{abstract}

\section{INTRODUCTION}

Potential applications of optical wavelength-selective structures, such as bio-sensing, spectral analysis, routing (switching) in optical communication networks have attracted many research efforts from a wide scientific community. Photonic crystals (PCs) are perfect candidates to construct such devices. Due to the unique guiding mechanism of PCs, an electromagnetic wave inside a PC structure can be prohibited to propagate at certain frequencies and in certain directions within the structure. Various approaches have taken advantage of this property for in wavelength-splitting, such as directional couplers [1, 2], micro-cavities [3, 4], graded index structures [5-7] or even semiconductor nano-wires [8]. While many of those approaches successfully implemented the idea of wavelength de-multiplexing, there is still the need for efficient and compact designs with very low cross-talk between channels.

A wave propagating in a photonic crystal waveguide (PCWG) can be substantially slowed down and localized, by the band-gap effect. This phenomenon is also known as slow light and can be achieved for different wavelengths by providing different local band-gaps at different locations on the PCWG, so that certain frequencies can propagate only at certain regions in the PCWG. One way to construct a PCWG with different local band-gaps is to gradually change the width of the waveguide. It is known that, decreasing the PCWG width increases the effective index of the guided mode and shifts its dispersion curve down in the band structure. The change in the dispersion curve, introduces new frequencies that will be located in the band-gap area. Thus, a propagating beam can be allowed to propagate in a PCWG with a wider waveguide, and can be inhibited to propagate in a narrower waveguide. Consequently, the cut-off region of a guided mode (boundaries of the transmission band) can be engineered so that the width modulated waveguide acts as an optical filter.

\section{DESIGN APPROACH AND NUMERICAL RESULTS}

In this study, we demonstrate that a tapered PCWG can localize and demultiplex a propagating electromagnetic wave at various locations. As illustrated in Fig. 1, a two-dimensional (2D) square lattice PC is designed with an angled line defect that forms a tapered waveguide with a tip angle, $\theta$. The line defect with an angle separates the structure into two symmetric PC regions, which are tilted up and tilted down by an angle of $\theta / 2$. The PC regions consists of Silicon (Si) rods, with a refractive index of $n=3.46$ and a radius equal to $r=0.35 a$, where $a$ is the lattice constant. The structural length and height of both PC regions are set to $L_{x}=50 a$ and $L_{y}=10 a$ as shown in Fig. 1, respectively.

To better understand the localization and trapping mechanism created by the tapered PCWG, a continuous wave with transverse magnetic (TM) polarization was launched into the input of the PCWG. To determine the frequency response of the WG structure the plane wave expansion method is used. The regarding PC super-cell columns of the tapered waveguide is exploited to calculate the dispersion diagram. We should note that, the operating frequencies are selected in band regions where slow light effects can be observed. Next, finitedifference time domain (FDTD) method in 2D [9] was employed for the calculation of the spatial electric-field 
intensity distribution at the operating frequencies $a / \lambda=\{0.39,0.40,0.41,0.42\}$. In this case a tapered PCWG with tip angle equal to $\theta=0.40^{\circ}$ is used and the corresponding field intensity distributions are given in Figs. 2(a), 2(b), 2(c) and 2(d), respectively. Moreover, the dependency of the operating frequency on the localization point is plotted in Fig. 2(e), to show the relationship more quantitatively. One can deduce that the relationship between the operating frequency and the travelling distance to get localized light is nearly linear. Hence, one can easily calculate the stopping point analytically, for the other frequencies within the operational bandwidth. This is also useful if one needs to select an optimum structural length $L_{x}$, according to a desired operational bandwidth.

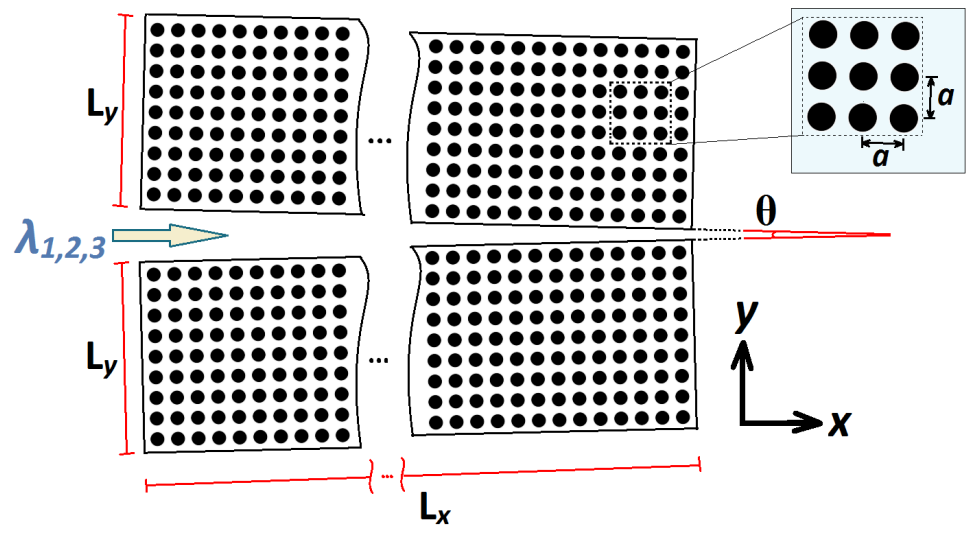

Figure 1. Schematics of the tapered photonic crystal waveguide with structural parameters $L_{x}$ and $L_{y}$. The zoomed version of the selected PC square lattice region is depicted as an inset.

After we determine the response of the tapered waveguide to the selected frequencies, transverse line defects as drop channels in the perpendicular direction were introduced as depicted in Fig. 3(a). The channel separations have distance values equal to $L_{C H 1}=8 a, L_{C H 2}=18 a, L_{C H 3}=28 a$ with respect to the input plane. These channels, denoted as $\mathrm{CH} 1, \mathrm{CH} 2$ and $\mathrm{CH} 3$, are expected to couple the localized light on the main channel and guide it towards the drop channels. To test this expectation, the transmission efficiencies of the drop channels $\mathrm{CH} 1, \mathrm{CH} 2$ and $\mathrm{CH} 3$ are numerically calculated. For this reason, a Gaussian pulse is launched into the waveguide. Detectors are placed at the end side of the drop channels. The transmission efficiencies are calculated and normalized by dividing the detected power to the incident power. The regarding results for the tapered $\mathrm{PCWG}$, with $\theta=0.40^{\circ}$ and $\theta=0.80^{\circ}$ are given in Figs. 3(b) and 3(c), respectively. Figures 3(b) and 3(c), indicate distinct peaks in the transmission spectra, as expected.
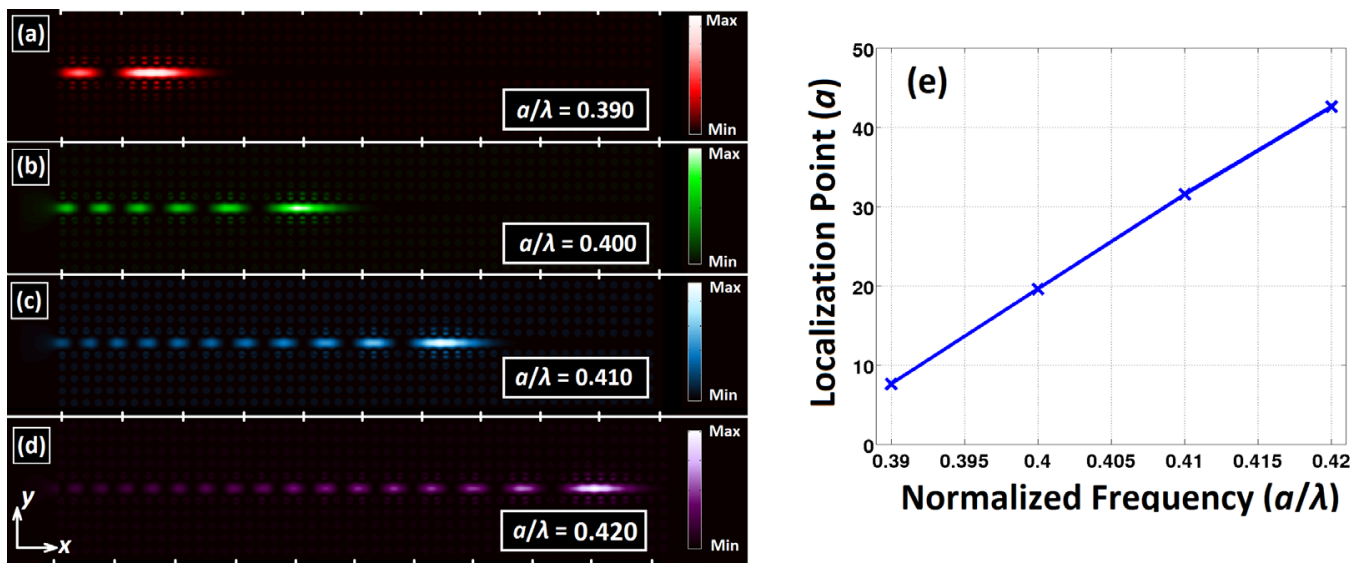

Figure 2. Spatial electric-field intensity distributions of the tapered PCWG operating at normalized frequencies (a) a/ $\lambda=0.39$, (b) $a / \lambda=0.40$, (c) $a / \lambda=0.41$ and (d) $a / \lambda=0.42$. (e) The localization points where each frequency gets trapped.

However, sidelobes (local peaks near the expected frequency) with relatively large amplitudes seem to cause crosstalk and loss in the dropping efficiency. These preliminary results encourage us to continue for further optimization about the determining the locations of dropping channels and enhancing the effective wavelength selectivity. The study continues considering these aspects of the problem.

For further inspection, the tapered PCWG with drop channels is illuminated by a continuous source with frequencies obtained from the peaks of the transmission spectra. These frequencies correspond to $a / \lambda=\{0.390$, $0.398,0.407\}$ and $a / \lambda=\{0.402,0.419,0.435\}$ for $\theta=0.40^{\circ}$ and $\theta=0.80^{\circ}$, respectively. Figures $4(\mathrm{a}-\mathrm{b}-\mathrm{c})$ and Figs. 4(d-e-f) demonstrate the steady state field distributions at these peak frequencies, for the two inclined 
angles: $\theta=0.40^{\circ}$ and $\theta=0.80^{\circ}$, respectively. Simulation results show that, the selected frequencies are coupled into the drop channels. However, crosstalks appear at both configurations, as expected.
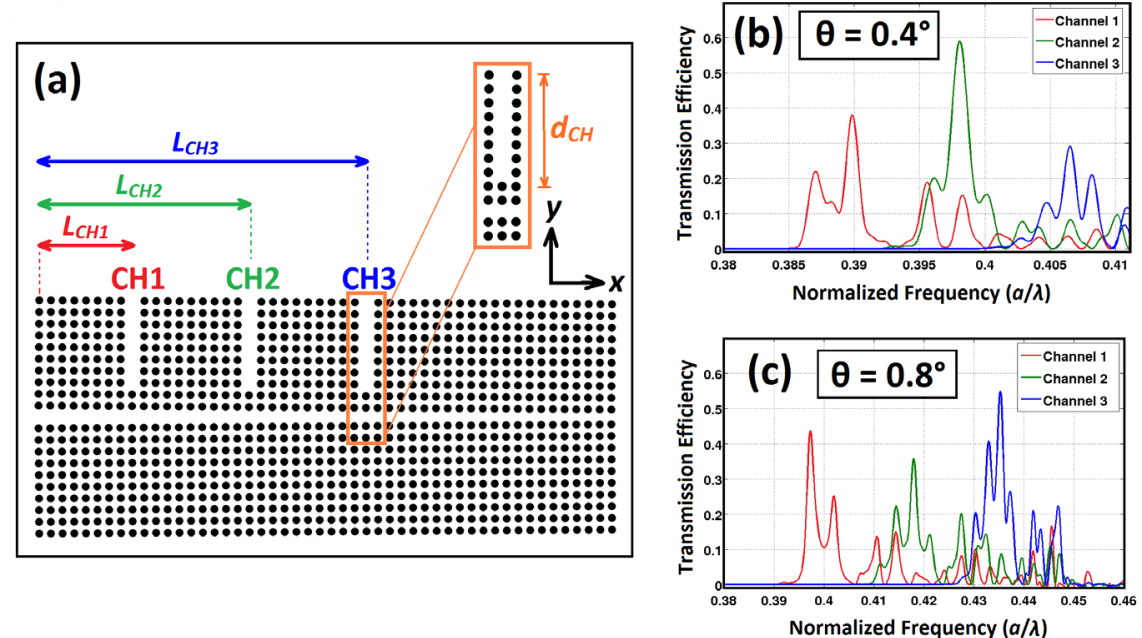

Figure 3: (a) Schematics of the tapered photonic crystal waveguide with drop channels . The heights of the channels are equal to $d_{C H}=8 a$. The transmission spectrums obtained at the drop channels are given for (b) $\theta=0.4^{\circ}$ and (c) $\theta=0.8^{\circ}$.

The existence of the leakages (cross-talks) can be prohibited. In order to tackle the problem, the separations of the drop channels become important. Besides, after light localizes at different positions, the dropping efficiency can be manipulated by optimizing the coupling strategy between horizontal and vertical waveguides. In the preliminary result, we keep only two dielectric rods without altering their locations or radii. As a result, there will be improvement for the dropping efficiency if one modifies opto-geometric values of the coupling region.

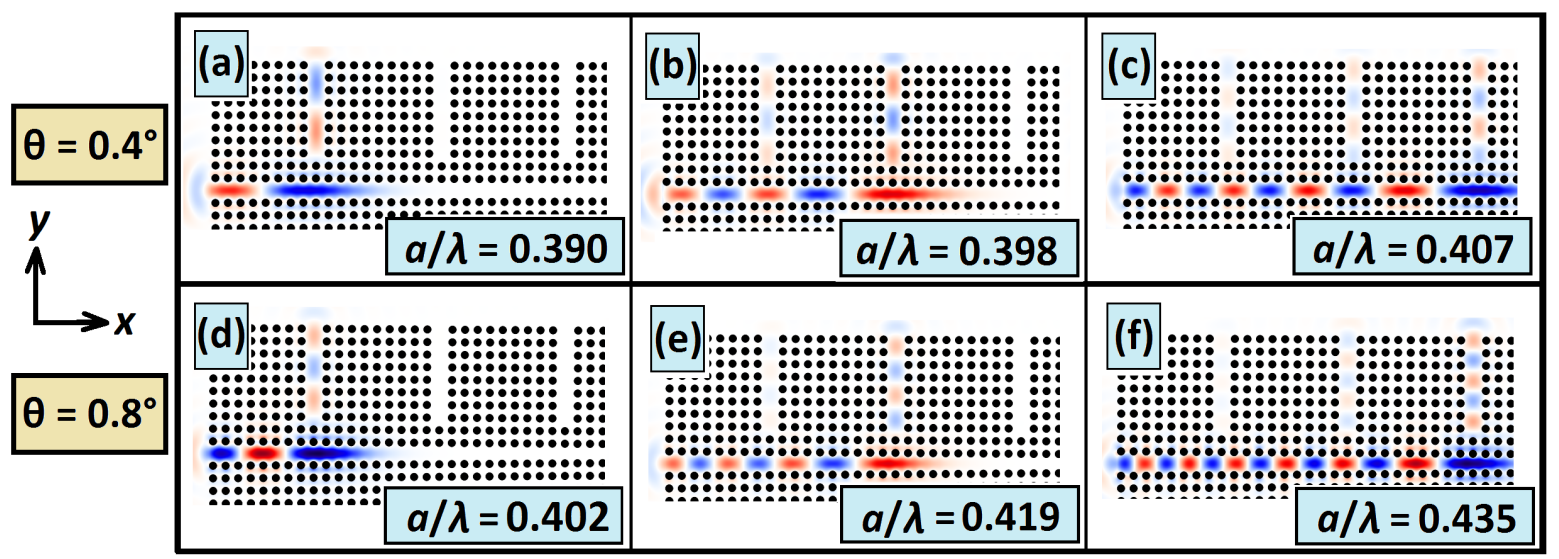

Figure 4. Steady state field distributions of the tapered $P C W G$ with $\theta=0.40^{\circ}$ and operating frequencies (a) $a / \lambda=0.390$, (b) $a / \lambda=0.398$ and (c) $a / \lambda=0.407$; with $\theta=0.80^{\circ}$ and operating frequencies $(d) a / \lambda=0.397$, (e) $a / \lambda=0.419$ and $(f) a / \lambda=0.435$.

\section{CONCLUSIONS}

The light localization and demultiplexing abilities of a tapered photonic crystal waveguide is proposed and numerically verified. Calculated spectra via the FDTD method, confirms the operational principal. Moreover, the designed structure operates in a linear region, which means there is a linear relation between drop frequencies and positions. The results also verify the tunability of the structure. Different inclination angles produces different waveguide filter acting as de-multiplexer with the placement of drop channels. Both the simplicity of the design and the linear operating ability are the major advantageous of the designed wavelengthdemultiplexer. However, in terms of cross-talks and efficiency losses, the designed structure demands for further improvements.

\section{ACKNOWLEDGEMENTS}

H.K. acknowledges partial support of the Turkish Academy of Science. 


\section{REFERENCES}

[1] J. Zimmermann, M. Kamp, A. Forchel, and R. Marz: Photonic crystal waveguide directional couplers as wavelength selective optical filters, Optics Communications, 230, 387-392 (2004).

[2] S. Boscolo, M. Midrio, and C. G. Someda: Coupling and decoupling of electromagnetic waves in parallel 2D photonic crystal waveguides, IEEE Journal of Quantum Electronics, 38, 47-53 (2002).

[3] H. Ren, C. Jiang, W. Hu, M. Gao, and J. Wang: Photonic crystal channel drop filter with a wavelengthselective reflection micro-cavity, Optics Express, 14, 2446-2458 (2006).

[4] D. Paciotti, A. Marchese, M. S. Moreolo, and G. Cincotti: 2D photonic crystal cavity-based WDM multiplexer, Photonics and Nanostructures-Fundamentals and Applications, 5, 164-170 (2007).

[5] H. Kurt and D. Yilmaz: Rainbow trapping using chirped all-dielectric periodic structures, Applied Physics B, 110, 411-417 (2013).

[6] D. Yilmaz, I. H. Giden, M. Turduev, and H. Kurt: Design of wavelength selective medium by graded index photonic crystals, IEEE Journal of Quantum Electronics, 49, 477-484 (2013).

[7] A.E. Akosman, M. Mutlu, H. Kurt, and E. Ozbay: Compact wavelength de-multiplexer design using slow light regime of photonic crystal waveguides, Optics Express, 19, 24129-24138 (2011).

[8] H.G. Park, C.J. Barrelet, Y. Wu, B. Tian, F. Qian, and C.M. Lieber: A wavelength-selective photonic-crystal waveguide coupled to a nanowire light source, Nature Photonics, 2, 622-626 (2008).

[9] A.F. Oskooi, D. Roundy, M. Ibanescu, P. Bermel, J.D. Joannopoulos, and S.G. Johnson: MEEP: A flexible free-software package for electromagnetic simulations by the FDTD method", Computer Physics Communications, 181, 687-702 (2010). 